\title{
Frontières
}

\section{Au-delà des mots pour dire la peur, être capable de bonté pour dire la vie}

\section{Diane Laflamme}

Volume 12, numéro 2, printemps 2000

Peur bleue...

URI : https://id.erudit.org/iderudit/1074392ar

DOI : https://doi.org/10.7202/1074392ar

Aller au sommaire du numéro

Éditeur(s)

Université du Québec à Montréal

ISSN

1180-3479 (imprimé)

1916-0976 (numérique)

Découvrir la revue

Citer ce document

Laflamme, D. (2000). Au-delà des mots pour dire la peur, être capable de bonté pour dire la vie. Frontières, 12(2), 10-13. https://doi.org/10.7202/1074392ar d'utilisation que vous pouvez consulter en ligne.

https://apropos.erudit.org/fr/usagers/politique-dutilisation/ 


\section{P R É S E N T A T I O O N}

\section{Au-delà des mots pour dire la peur, être capable de bonté pour dire la vie}

Diane Laflamme,

coresponsable du numéro sur la Peur, doctorante en Sciences humaines appliquées à l'Université de Montréal.

Quand nous avons mis sur le métier ce numéro de Frontières, nous tous qui en avons placé les fils de chaîne et les fils de trame, nous savions que les mots servant à tisser le thème de la peur se bousculeraient sous la navette. Dans l'ouvrage achevé, tous ces mots ont effectivement pris leur place, formant des dessins, obéissant à des desseins... se répondant l'un à l'autre. Je vous propose ici de les regarder de plus près avant de commencer la lecture des articles. Je les aborde en m'attardant à trois motifs. Dans un coin de l'ouvrage, j'écris à mon tour le mot bonté.

\section{LA PEUR BLEUE: UNE PEUR INTENSE}

Comme première référence pour situer tous ces mots qui disent la peur, je m'en tiens à un outil à la portée de tout le monde: Le Petit Robert. Au risque de faire sourire les habitués des savantes bibliographies, je vois là une façon d'éclairer en lumière blanche les concepts que chacune et chacun de nos auteurs mettent en scène sous des éclairages différents, selon le point de vue qu'ils choisissent de privilégier. Voici le lexique qui se tisse alors devant nos yeux, autour de la peur bleue et des phénomènes reliés à la peur.

\section{PREMIER MOTIF DU TISSAGE: \\ DE LA CRAINTE À L'HORREUR}

À mesure que la peur commence à mieux dire son nom, on se retrouve presque imperceptiblement en train de tourner en rond dans le dictionnaire. Les définitions de tous ces mots que nous avons inventés en français autour de la peur s'inscrivent dans une circularité incon- tournable puisqu'elle tient à la nature même du processus qui nous donne les mots de cette langue que nous parlons... et qui nous parle, comme le dit la psychanalyse depuis Lacan.

Commençons ce premier motif avec un cercle de six mots, de la crainte à l'horreur, à mesure que le premier mot nous renvoie à un autre, puis à un autre encore...

Crainte: "(XIIIe siècle) sentiment par lequel on craint (quelque chose ou quelqu'un); appréhension inquiète.»

Phobie: "(1880) 1. Psychol.: crainte excessive, maladive et irraisonnée de certains objets, actes, situations ou idées. 2. Courant: peur ou aversion instinctive.»

Épouvante: «(1570) 1. peur violente et soudaine causée par quelque chose d'extraordinaire, de menaçant. 2. Par extension: vive inquiétude.»

Effroi: "(1140) Littér.: grande frayeur, souvent mêlée d'horreur, qui glace, qui saisit.»

Frayeur: "(1460) peur très vive, généralement passagère, provoquée par un danger réel ou supposé.»

Horreur: "(1160) Sens subjectif: impression violente causée par la vue ou la pensée d'une chose affreuse ou repoussante. Sens objectif: Caractère de ce qui inspire ou peut inspirer de l'effroi, de la répulsion.»

Ce parcours circulaire pour nommer la peur n'est pas un jeu aussi anodin qu'il en a l'air. Nous en sommes avertis grâce au "Regard» lucide que porte Réjane Charpentier, une "phraseuse» qui n'a pas peur des mots. Comme elle le décrit si bien, il y aura combat: "La peur est notre ange de Jacob. Ou bien nous acceptons de combattre avec elle jusqu'à ce qu'elle dévoile son nom ou bien elle nous rongera la peau jusqu'à l'os". Face à cet ange, un simple réflexe de combat pour la survie du plus fort ou du mieux adapté ne suffira pas. Face à cet ange, nous découvrirons aussi qu'il devra y avoir réflexion, travail, afin que la pensée sur la peur enri- 
chisse le réflexe qui se déclenche en face d'elle, comme le dit Luce Des Aulniers dans son article sur la quête identitaire comme peur de l'autre.

Les définitions du dictionnaire nous permettent de constater comment tous ces mots qui disent la peur sont apparus progressivement au cours des siècles. Plusieurs de nos auteurs ont aussi jugé utile de reculer dans le temps pour mieux nous parler de la peur. Dans les sections Article et Note de recherche, les citations de Jean Delumeau et de Georges Duby fournissent des points de repère pour ce voyage dans le temps. Les fouilles menées par Duby pour documenter la genèse des peurs dans l'histoire des mentalités nous ramènent au tournant du premier millénaire de la chrétienté, alors qu'il nous faut à notre tour prendre le tournant vers un deuxième millénaire; l'entretien avec Delumeau cherche des balises pour comprendre le phénomène de la culpabilisation en Occident, faisant référence à des recherches portant sur les années entre 1348 et 1648 . Selon Delumeau, nous n'avons pas à "rougir d'avoir peur» encore aujourd'hui. Dans la section Regard, Léo Rosshandler choisit de faire référence à un manuscrit du XVe siècle, trouvant là une illustration des liens imprévus qui peuvent relier, souvent même à l'insu du créateur lui-même, la peur et l'art. Dans la section Point de vue, Denis Jeffrey nous propose un itinéraire surprenant alors qu'il suit à la trace, à travers diverses mythologies, la peur des loups. Toujours dans Point de vue, Nathalie Ferrand nous ramène enfin aux lieux et temps que nous habitons aujourd'hui dans nos villes: elle a choisi comme interlocuteurs, pour aborder la peur du feu, des pompiers rencontrés dans une caserne de quartier, à Montréal.

\section{DEUXIĖME MOTIF DU TISSAGE: PEUR ET ANGOISSE}

Dans la ronde des mots, il y a aussi des couplages qui attirent le regard, qui font ressortir des contrastes. En voici un premier:

Peur: “(1290) Sens fort: phénomène psychologique à caractère affectif marqué, qui accompagne la prise de conscience d'un danger réel ou imaginé, d'une menace. Voir: affolement, alarme; alerte, angoisse, appréhension, crainte, effroi, épouvante, frayeur, inquiétude, panique, terreur; fam.: frousse, trouille. [...] hantise $[\ldots]$ aversion, phobie, répulsion.»

Cette distinction entre peur et angoisse, plusieurs de nos auteurs l'abordent en détail et en proposent une cartographie plus précise à partir de concepts tirés des champs d'études où ils se spécialisent ou à partir de l'expérience vécue. Ainsi l'entrevue avec Isabelle Lasvergnas-Grémy contient des passages expliquant le point de vue de la psychanalyse, selon laquelle les deux principales angoisses sont l'angoisse de castration et l'angoisse de mort. À côté de l'état d'angoisse, qu'elle

présente comme "un état pulsionnel au plus proche d'un ressenti", Isabelle Lasvergnas-Grémy rappelle, reprenant cette fois le vocabulaire de la sociologie, que la peur a une forme, un contenu, et que le discours de la peur est culturellement codé: «il reflète l'esprit du temps auquel il appartient». C'est d'ailleurs ce qui le «rend audible pour les autres». Isabelle Lasvergnas-Grémy ajoute qu'il n'y a pas lieu de fuir l'angoisse à tout prix et signale la possibilité d'un travail sur soi qui serait non seulement dépassement de l'angoisse mais création.

L'angoisse et même l'angoissement, terme qu'il trouve chez Paul Diel pour désigner l'ensemble des formes d'angoisse, font partie des balises que se donne Pierre Migneault pour en arriver à "rendre son dû à la peur». Dans son article, peur, angoisse et désarroi sont abordés comme un triptyque, même si le noyau dur reste la peur tout court. C'est du texte de Pierre Migneault que je tire et je retiens la très belle expression de "peur-vigie». La vigilance permet à la vie de continuer à cheminer, en côtoyant "peur et émerveillement».

Avec l'angoisse, la thématique de la mort commence à être plus précisément nommée par nos auteurs. Les "parcours passionnels" de Christiane Kègle dessinent par petites touches juxtaposées peur et angoisse de mort, vécues dans l'irremplaçable d'une histoire personnelle. Ces voix entremêlées ne cherchent pas à définir ce que le dictionnaire qualifie de phénomène psychologique ou de malaise; elles racontent plutôt comment s'alourdit, au fil des mois et des saisons, le poids du mot angoisse.

\section{TROISIĖME MOTIF DU TISSAGE: ANXIÉTÉ ET MÊME TERREUR}

Le couplage suivant est peut-être celui où vie et mort tournent le plus furieusement en rond:

Anxiété: "(XIle siècle; repris en 1531) Méd.: état de trouble psychique causé par le sentiment de l'imminence d'un événement fâcheux ou dangereux, s'accompagnant souvent de phénomènes physiques.

Courant: État d'inquiétude extrême causé par l'appréhension d'un événement.»

C'est avec ce couple que nos auteurs cernent de plus près la thématique de la mort. Grâce à la Note de recherche de Lyne St-Amour et de Margaret C. Kiely, nous avons accès aux tentatives des chercheurs pour valider des échelles qui permettraient une mesure de l'anxiété relative à la mort: un sujet amplement documenté dans la littérature mais une observation difficile à faire en clinique. Leur texte se termine en évoquant, avec un point d'interrogation, la distinction à faire entre anxiété et angoisse: "L'anxiété pouvant référer à des manifestations plus observables alors que l'angoisse serait reliée à une douleur profonde liée à des tensions vitales?". Signalons au passage que le vocabulaire médico-psychiatrique, pour aborder les syndromes cliniques, s'en tient à ce qu'il est convenu d'appeler des troubles: les troubles anxieux, le trouble panique et la phobie. 


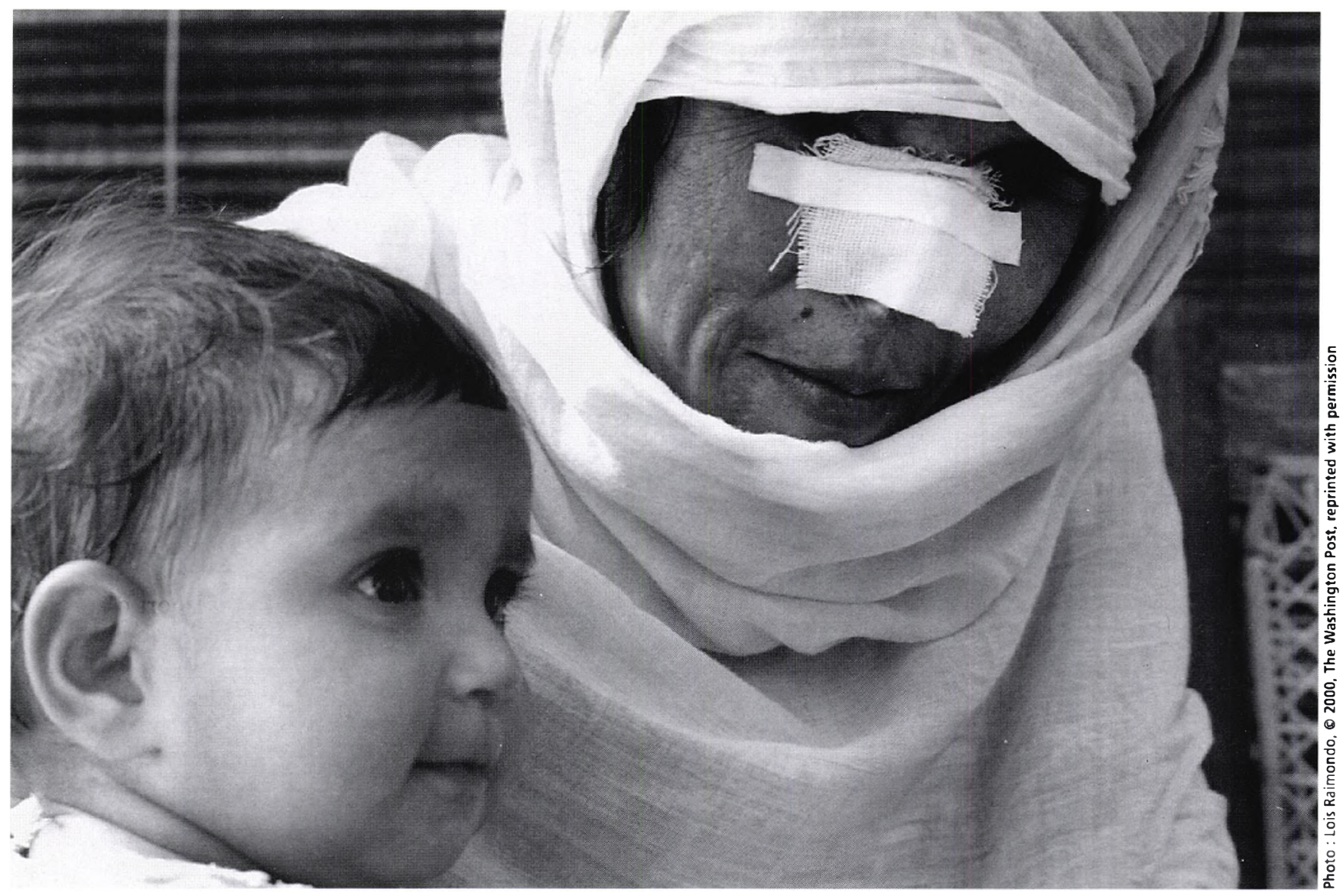

Wilfrid Noël Raby aborde aussi dans son article la notion d'anxiété, proposant des liens à faire avec la mémoire implicite et rappelant comment l'anxiété et la peur transforment le comportement de l'enfant et influencent son développement.

Raby fait ensuite la distinction entre peur et terreur: la peur est "un état d'être», dit-il, alors que la terreur "est produite par un acte, provoqué ou subi, devant lequel nous nous sentons englués d'impuissance, mais qui pourtant exige que nous réagissions». Il ajoute que l'«on peut légiférer ou s'armer contre la terreur, on ne peut pas le faire contre la peur». C'est aussi le mot terreur que Bernard J. Lapointe place dans le titre de son article, où il aborde la peur de la mort à partir de son expérience comme médecin oeuvrant en soins palliatifs. La piste qu'il explore dans son texte vient éclairer le rôle que pourrait jouer l'estime de soi face à la terreur de la mort. Lapointe se demande si la philosophie mise en place depuis la fin des années soixante par le mouvement des soins palliatifs pourrait compenser, ne seraitce que partiellement, ce qu'il appelle une lacune de cosmogonie signifiante et de valorisation qui serait le lot de plusieurs patients lorsqu'ils sont confrontés à l'imminence de leur propre mort. Poursuivant sur cette lancée, on trouvera peut-être pertinent d'envisager cet accompagnement dans «la compassion, la générosité et la réconciliation" comme un exercice de préparation à la bonne mort, dans la même lignée que les exercices proposés de tout temps par les traditions religieuses et philosophique. La note de recherche préparée à partir d'un article de Charles David Axelrod sur la peur de la mort peut alimenter une telle réflexion en mettant en évidence la logique sous-jacente aux exhortations à la préparation à la mort.
C'est avec le couple anxiété / terreur que font irruption dans notre propos la dimension politique et la dimension éthique, alors qu'on aurait pu vouloir s'en tenir à documenter des phénomènes d'introspection. Dans son article, Raby dit se donner pour tâche de montrer "comment les notions contemporaines de droits et de devoirs plongent leurs racines dans l'émotion, notamment la peur». Ce qu'il appelle ensuite le legs de la peur gravite autour du mot démocratie: "Le legs de la peur pour une démocratie mûrissante se consolide au fur et à mesure que se succèdent les générations pour qui les droits universels sont affirmés et protégés. À chaque génération, nos démocraties acquièrent en substance et en pesanteur un aplomb sans cesse accru pour affronter la terreur». Selon lui, "contenir la peur, en saisir le sens, se la remémorer, est absolument nécessaire à la sauvegarde de nos démocraties». La conclusion qui s'impose dès lors, Raby la formule clairement: "La peur nous conduit donc de plain pied à la façon dont coexistent le bien et le mal en notre monde».

\section{PEUR-VIGIE PARCE QUE NOUS AVONS RAISON D'AVOIR PEUR}

Raby parle de la peur "comme philtre à la fois tonique et poison». Les paroles de la chanson écrite par Sylvie Massicotte nous permettent de nous voir, de nous regarder aller quand nous sommes sous l'influence de ce philtre: «La mort fait peur. J'ai le corps en sueur. Pourtant j'avance». C'est ce que nous sommes, incarnés dans une chair, une culture, une époque, c'est ce qui a appris à parler pour dire Je, qui permet à la respiration de la vie de nous traverser ou de rester bloquée au travers de notre gorge. C'est ce que nous sommes, à mesure que nous le devenons, qui nous rend sensibles tant 
aux extases auxquelles la vie nous donne accès qu'aux horreurs auxquelles on peut consentir pour rester en vie.

Dans la dernière partie de son texte sur le legs de la peur, Raby rappelle et commente l'horrible scène du troisième acte du Roi Lear de Shakespeare où le Duc de Cornwall crève les yeux du Comte de Gloucester. Je connais une histoire semblable: celle de Zahida Perveen. Oserai-je vous la raconter? Car pour Zahida, la tragédie ne s'est pas passée au théâtre, elle a frappé à la maison, le 28 décembre 1998, alors qu'elle était enceinte.

Je ne connais Zahida Perveen que par cette photo publiée le 22 mai 2000 par le journal montréalais The Gazette, qui reprenait alors un reportage du Washington Post. C'est une femme de 32 ans. Elle habite à Gujar Khan, au Pakistan. En rentrant de la mosquée Mahmoud Iqbal, son mari, l'a accusée de l'avoir trompé avec un autre homme et d'avoir mauvais caractère. Il a quarante ans. Il est barbier. Avec un rasoir et un couteau, il l'a défigurée: il lui a crevé les yeux, il lui a coupé le nez et les oreilles. Il dit que c'était une question d'honneur. Maintenant, il se dit satisfait.

Oserai-je vous dire ma peur en apprenant cette histoire et en lisant les statistiques complémentaires qui figuraient aussi dans l'article? Terreur, face au pouvoir totalitaire. Il faut pourtant continuer de nourrir la vie, d'en prendre bien soin: c'est ce que je me dis quand je regarde cette photo de Zahira avec son enfant. Nous sommes "capables" de prendre soin de la vie, tous ensemble. Nous n'avons pas à avoir peur car, pour cette tâche, nous ne sommes pas irrémédiablement seuls, chacun pour soi. Paradoxalement, c'est aussi parce que nous ne sommes pas seuls que nous avons raison d'avoir peur. Ici encore les mots se mettent rapidement à faire la ronde...

S'il existe un antidote à la peur, c'est peut-être la bonté. Celles et ceux qui oeuvrent en soins palliatifs le constatent tous les jours et pourraient en donner maints exemples. La bonté inspire rarement de grands discours. Elle se sait en travail, vulnérable aux erreurs, facilement aveuglée. Elle se dit dans le quotidien du monde ordinaire. Pour en parler la poésie se sert de mots simples, qui avancent lentement avant de faire la ronde eux aussi, comme le font les enfants quand on les laisse jouer dehors:

«Il faut plus d'une pomme

Pour emplir un panier.

Il faut plus d'un pommier

Pour que chante un verger.

Mais il ne faut qu'un homme

Pour qu'un peu de bonté

Luise comme une pomme

Que l'on va partager.»

Maurice Carême, La Lanterne magique, Bourrelier et Colin, Paris, 1947.

\section{J'AVANCE}

Le chocolat

Qui fond entre mes doigts

J'ai froid, j'ai chaud

Pendant qu'ils parlent trop

Ils me serrent fort

Me racontent que tu dors

La mort fait peur

J'ai le corps en sueur

Pourtant j'avance

Ils ont croisé tes mains blanches et glacées

Moi, j'ai sali mes doigts

Mes manches aussi

Le chocolat

Les choses d'ici-bas

Perdent leur goût

Quand tu n'es pas debout

Et que j'avance

Je fixe sans les voir

Mes chaussures noires

Et j'avance, j'avance

J'avance sans y croire

J'avance sans y croire

N'entends-tu pas

Ce qu'ils disent tout bas

Change-moi la fin

Ton histoire n'est pas bien

Tu n'es pas drôle

Sous cette gerbe de roses

J'compte jusqu'à trois

Sors de ce coffre en bois

Pour que j'avance.

Paroles de Sylvie Massicotte

Musique de Breen Lebœuf

Tiré du disque "J'avance» de Breen Lebœuf

Productions 2000. 